\title{
Le système nerveux dans le programme de biologie : quel(s) concept(s) veut-on enseigner?
}

Jean-Louis Bec et Daniel Favre

\section{OpenEdition}

Édition électronique

URL : http://journals.openedition.org/trema/2039

DOI : 10.4000/trema.2039

ISSN : 2107-0997

Éditeur

Faculté d'Éducation de l'université de Montpellier

\section{Édition imprimée}

Date de publication : 1 septembre 1996

Pagination : 97-104

ISSN : 1167-315X

\section{Référence électronique}

Jean-Louis Bec et Daniel Favre, "Le système nerveux dans le programme de biologie : quel(s) concept

(s) veut-on enseigner? », Tréma [En ligne], 9-10 | 1996, mis en ligne le 18 septembre 2013, consulté le 30 avril 2019. URL : http://journals.openedition.org/trema/2039 ; DOI : 10.4000/trema.2039

Ce document a été généré automatiquement le 30 avril 2019.

Trema 


\title{
Le système nerveux dans le programme de biologie : quel(s) concept(s) veut-on enseigner?
}

\author{
Jean-Louis Bec et Daniel Favre
}

\section{Introduction}

1 Parmi l'ensemble des concepts utilisés en Didactique, celui dont le succès semble être le plus important ces dix dernières années est celui de conception. Il est couramment admis maintenant que tout apprentissage viendrait interférer chez l'apprenant avec un déjà-là conceptuel, les conceptions, qui constituent pour lui un système d'explication lui permettant de résoudre ses problèmes. Un apprentissage effectivement réalisé par l'élève impliquerait un remaniement, une transformation durable de ses conceptions. Ainsi, une démarche beaucoup utilisée en Didactique pour vérifier l'intégration de nouvelles connaissances consiste à tenter d'inférer les conceptions des élèves avant et après la séquence d'apprentissage, ceci afin de pouvoir établir le bilan de leurs modifications.

2 Nous nous sommes attachés à suivre cette démarche pour évaluer l'impact que représentait le cours de Biologie traitant du système nerveux sur l'ensemble des conceptions que possédaient les élèves de Terminale au sujet de certains concepts qui sont introduits, définis lors de cet enseignement en classe de Seconde et sur lesquels s'appuie également le contenu du programme de Terminale. Le fait que nous nous soyons intéressés à la Didactique du système nerveux a été motivé par le fait que celle-ci a suscité encore peu de travaux de recherche (Clément ; 1993, 1994), (Darley; 1994), (MAFPEN ; 1994), (Mein ; 1988), (Mein et Clément ; 1988) et qu'elle restait donc un domaine encore largement inexploré comparativement à celle de la respiration ou de la digestion. Le fait d'inférer les conceptions des élèves de Terminale a été plus spécifiquement motivé, lui, par la nature des résultats obtenus par Darley (1994). Celui-ci précise en effet dans son étude que les étudiants de Deug B 2e année commettent de nombreuses confusions quand 
ils ont à définir ce qu'est le potentiel d'action. Au vu de cela, il est logique de penser que ces erreurs relevées au niveau de l'Enseignement Supérieur ont pu se construire au cours des années précédentes, à la suite des enseignements survenus au niveau des classes de Seconde et de Terminale. Il est également envisageable que si les élèves ont rencontré des difficultés pour acquérir le concept de potentiel d'action tel que celui-ci est introduit, défini au cours de l'enseignement qui traite du système nerveux au lycée, Os en ont rencontré également pour assimiler les autres concepts introduits au cours de ce même enseignement.

3 L'objectif qui nous a poussés à réaliser cette étude a donc été de déterminer si les élèves parviennent à acquérir les concepts tels qu'Os sont définis au cours des séances d'enseignement qui traitent du système nerveux au lycée.

4 Un point nous a semblé important à préciser dans le cadre de cette recherche. C'est de déterminer si la nature des conceptions des élèves varie en fonction du type de leur formation, scientifique ou littéraire.

\section{Méthode adoptée pour inférer les conceptions des élèves de Terminale}

Dans un premier temps, nous avons examiné le contenu du programme de Seconde tel que celui-ci est défini par le texte du Bulletin Officiel du 24 septembre 1992. C'est en effet ce programme que les élèves se trouvant en Terminale au cours de l'année 94-95 ont connu en classe de Seconde. Nous avons également consulté le contenu du cours proposé par un manuel scolaire utilisé en classe de Seconde (collection Tavernier, Bordas, 1992), ceci afin de repérer, d'identifier les concepts dont les définitions apparaissaient nettement au cours de l'enseignement relatif au système nerveux. La présence des concepts de stimulus, de récepteur, de message nerveux, de codage de l'information, de réflexe et de présence de messages nerveux dans la remémoration a ainsi été relevée.

6 Nous avons alors inféré les conceptions que des élèves d'une classe de Terminale L (TL) et de 2 classes Terminale $S$ de spécialité Biologie (TSbio) et de spécialité Mathématiques (TSmath) avaient développées au sujet de ces concepts à l'aide de deux questionnaires semblables, le premier étant proposé aux élèves avant le cours de Terminale traitant du système nerveux, le second après.

7 Afin de déterminer si le fait de répondre au premier questionnaire pouvait influencer la nature des réponses données au deuxième questionnaire par les élèves, et ceci indépendamment de tout effet d'apprentissage dû au cours, nous avons également étudié les conceptions que possédaient les élèves d'une classe de Terminale ES (TES) dont la formation scolaire ne prévoit pas d'enseignement de Biologie. Cette classe nous a donc servi de classe témoin tout au long de notre expérimentation. L'analyse des réponses données par ces élèves aux deux questionnaires n'a pas révélé de modification importante de leurs conceptions. 


\section{Conceptions des élèves de Terminale inférées avant et après le cours traitant du système nerveux}

8 L'analyse qualitative et quantitative par concept et par classe des réponses obtenues au premier puis au second questionnaire a permis d'inférer les conceptions suivantes.

\subsection{Conceptions développées par les élèves au sujet du stimulus et du récepteur sensoriel avant et après le cours}

9 Dans le cours de Seconde, le stimulus est défini comme étant un phénomène extérieur qui agit sur les récepteurs sensoriels de manière efficace. Ces derniers génèrent alors des messages nerveux qui sont par la suite intégrés par un centre nerveux. La spécificité et la sensibilité vis-à-vis du stimulus sont les propriétés essentielles du récepteur sensoriel.

10 Si la majorité des élèves de TSmath et TSbio ( $71 \%$ et $93 \%$ respectivement) a atteint au sujet du stimulus un degré de conceptualisation qui associe effectivement celui-ci à un phénomène extérieur, la majorité des élèves des classes de Terminale L et ES, (55\% et $52 \%$ respectivement), n'y est, par contre, pas parvenue. Il est à noter également que parmi les élèves de TS qui attribuent au stimulus la définition de phénomène extérieur, $72 \%$ et $65 \%$ des élèves de TSmath et TSbio respectivement, limitent l'effet du stimulus à une action sur les sens, sans donner de précision biologique supplémentaire sur la réalisation de ces phénomènes. Ainsi, aucun élève ne mentionne où se trouve le récepteur sensoriel, la génération du message nerveux.

11 L'analyse des réponses que les élèves ont données au deuxième questionnaire révèle que le pourcentage des élèves de chacune des classes qui ont atteint un niveau de conceptualisation leur permettant d'attribuer au stimulus une action sur les sens a augmenté de $6 \%, 17 \%, 20 \%$ dans les classes de TL, TSmath et TSbio respectivement. Ces augmentations sont du même ordre si l'on considère le fait de citer le récepteur sensoriel et celui de signaler la génération du message nerveux sous l'effet du stimulus. Toutefois, le fait de rechercher plus en profondeur quelles sont les propriétés que les élèves attribuent au récepteur sensoriel permet de mettre en évidence chez les élèves, la présence d'obstacles ou de lacunes qui sont préjudiciables à l'assimilation de ce concept tel qu'il est défini dans le cours. Seulement $6 \%$ et $25 \%$ de l'ensemble des élèves des classes de TL et de TSbio respectivement signalent de façon conjointe la sensibilité et la sélectivité comme étant les propriétés essentielles du récepteur. Le reste des élèves ne considère soit aucune de ces propriétés, soit n'en cite qu'une.

\subsection{Conceptions développées par les élèves au sujet de la nature du message nerveux avant et après le cours}

Dans le cours de Seconde, il est spécifié que le message nerveux est un des supports de l'information qui circule au sein de l'organisme pour assurer la communication entre ses différents organes. Il est généré, soit par les organes sensoriels sous l'effet de stimuli, soit par les centres nerveux. Il est spécifié également que le message nerveux se présente sous la forme de signaux élémentaires de nature électrique, mais utilise également des supports chimiques au niveau des synapses. 
13 De l'étude des réponses données au premier questionnaire, il résulte que la majorité des élèves des quatre classes attribue au message nerveux le statut d'information. C'est le cas de $72 \%, 69 \%, 85 \%$ et $90 \%$ des élèves des classes de TES, TL, TSmath et TSbio respectivement. Mais, sur l'ensemble des élèves de chacune des classes, on constate que $50 \%, 40 \%, 45 \%$ et $50 \%$ d'entre eux limitent le trajet suivi par cette information à l'axe cerveau/corps sans donner de précision sur la nature du message nerveux. Il est à noter également que sur l'ensemble des élèves de chacune des classes, seulement 3,5\% (1 élève), $14 \%$ et $19 \%$ des élèves de TL, TSmath et TSbio respectivement considèrent que le message nerveux est de nature électrique et qu'il peut se déplacer sur l'ensemble du réseau nerveux. Aucun élève ne signale l'existence de la synapse.

cours traitant du système nerveux, le pourcentage des élèves associant le message nerveux à une information a augmenté de $5 \%, 10 \%$ et $10 \%$ dans les classes de TL, TSmath et TSbio respectivement. Le pourcentage des élèves considérant la nature électrique du message nerveux tout en ne limitant pas son trajet à l'axe cerveau/corps a également augmenté. Cette augmentation est de $4 \%, 28 \%$ et $11 \%$ dans les classes de TL, TSmath et TSbio respectivement. Toutefois, il faut noter que $48 \%, 63 \%$ et $50 \%$ des élèves des classes de TL, TSmath et TSbio respectivement limitent le message nerveux à l'axe cerveau/corps, que celui-ci ait été défini comme une simple information ou comme ayant une nature électrique.

Il est à noter également que seulement $5 \%$ des élèves de la classe de TS-math ont signalé un mode de propagation chimique du message nerveux. Aucun élève des autres classes ne l'a signalé.

\subsection{Conceptions développées par les élèves au sujet de la nature du codage de l'information avant et après le cours}

Dans le cours de Seconde, il est spécifié que l'information est codée sous la forme d'un message nerveux constitué par une succession de signaux électriques de même amplitude. Plus l'intensité du stimulus est élevée, plus la fréquence des signaux est élevée. Tout message nerveux est donc codé en « modulation de fréquence ».

17 L'analyse des réponses des élèves révèle que sur l'ensemble des quatre classes considérées, aucun élève n'a véritablement acquis ce concept. Le niveau de conceptualisation qui se trouve être le plus approchant de la définition donnée ci-dessus s'identifie au fait de signaler une variation du message nerveux en fonction de l'intensité $\mathrm{du}$ stimulus, sans préciser toutefois en quoi réside cette variation, si elle est qualitative ou quantitative. Encore, cette conception n'est-elle présente que chez $17 \%, 26 \%$ et 3,5\% des élèves des classes de TL. TSmath et TSbio respectivement.

18 L'analyse des réponses obtenues pour le deuxième questionnaire montre, qu'après le cours de Terminale, ce concept n'est toujours pas assimilé par la majorité des élèves puisque seulement $14 \%$ des élèves des classes de TSmath et TSbio semblent le maîtriser.

\subsection{Conceptions développées par les élèves au sujet de la nature du réflexe avant et après le cours}

Dans le cours de Seconde est décrit l'arc réflexe : dans un premier temps un récepteur sensoriel donne naissance à un message nerveux sous l'effet d'un stimulus. Ce message 
nerveux transite ensuite le long d'un nerf sensitif jusqu'à un centre nerveux qui assure alors son intégration et répond en générant un nouveau message nerveux qui transite par un nerf moteur jusqu'à un effecteur. Suivant la nature du réflexe, le centre nerveux considéré peut être le cerveau ou la moelle épinière.

Lors de l'analyse des réponses que les élèves ont données au premier questionnaire, il apparait que $56 \%, 69 \%, 71 \%$ et $71 \%$ des élèves des classes de TES, TL, TSmath et TSbio signalent la présence de l'aller-retour du message nerveux entre le lieu de la stimulation et un centre nerveux. Mais parmi ces élèves $86 \%, 90 \%, 88 \%$ et $91 \%$ considèrent que la réalisation du réflexe fait obligatoirement intervenir le cerveau en tant que centre nerveux, sans considération pour la moelle épinière.

21 L'analyse des réponses données au deuxième questionnaire révèle que le pourcentage des élèves prenant en considération l'aller-retour du message nerveux et la sollicitation d'un centre nerveux dans le réflexe a augmenté de $4 \%, 4 \%$ et de $15 \%$ dans les classes de TL, TSmath et TSbio respectivement. Nous avons alors cherché à préciser l'influence du cours sur la nature des centres nerveux que ces élèves impliquent dans le réflexe. $26 \%, 65 \%$ et $83 \%$ des élèves des classes de TL, TSmath et TSbio respectivement qui ont signalé PAR du Message Nerveux considèrent à présent que le centre nerveux impliqué dans un réflexe survenant au niveau de la jambe est la moelle épinière. Mais, il est à noter également que $21 \%, 38 \%$ et $28 \%$ des élèves des classes de TL, TSmath et TSbio considèrent que la moelle épinière est le centre nerveux intervenant dans tous les réflexes, quels qu'ils soient.

\subsection{Conceptions développées par les élèves au sujet de la présence de messages nerveux dans la remémoration avant et après le cours}

Dans le cours de Seconde, il est spécifié que les centres nerveux assurent l'intégration des messages nerveux issus des récepteurs sensoriels, mais qu'ils peuvent générer euxmêmes de tels messages sans stimulation sensorielle. Une de leurs propriétés est de pouvoir conserver les "traces de leur histoire antérieure». Dans le cas de l'effort de mémoire, les neurones associés en réseaux qui sont présents au niveau du cerveau communiquent entre eux par l'émission de messages nerveux.

Si l'on considère les réponses que les élèves ont données au premier questionnaire, il apparait que l'ensemble des élèves ne conçoit la remémoration que s'il y a présence au préalable d'un stimulus sensoriel. Pour eux, les messages nerveux ne peuvent donc pas être générés par le cerveau seul et toute remémoration nécessite obligatoirement pour son déclenchement une stimulation sensorielle. Il est à noter que seulement $8 \%, 7 \%, 23 \%$ et $16 \%$ des élèves des classes de TES, TL, TSmath et TSbio respectivement mentionnent l'intervention des neurones du cortex cérébral lors de l'effort de mémoire.

Après le cours traitant du système nerveux, les élèves n'ont pas modifié leur conception au sujet de la présence de messages nerveux dans la remémoration. Ils considèrent toujours que tout acte de remémoration nécessite préalablement l'intervention des sens pour se réaliser. Le pourcentage d'élèves considérant l'activité neuronale dans la remémoration a augmenté de $10 \%, 30 \%$ dans les classes de TL et de TSbio, alors qu'il a reculé de $5 \%$ dans celle de TSmath. 


\section{Conclusion}

biologie traitant du système nerveux, nous avons cherché à préciser si le fait d'avoir reçu un tel enseignement leur avait permis d'acquérir cet ensemble de concepts qu'ils ne possédaient pas avant le cours. L'analyse des réponses données révèle que le cours a globalement un effet bénéfique mais limité sur l'appropriation des savoirs par les élèves de Terminale. Il ressort en effet de cette étude que les concepts définis dans le programme de Seconde, auxquels nous nous sommes intéressés ici, ne sont toujours pas assimilés par la majorité des élèves. C'est le cas des concepts de messages nerveux, de codage de l'information, de récepteur sensoriel et de présence de messages nerveux dans la remémoration. En effet, il ressort de notre étude que la majorité des élèves n'a toujours pas développé l'étape d'abstraction nécessaire pour concevoir que le parcours suivi par le message nerveux n'est pas limité à l'axe cerveau/corps ou qu'il est possible de se remémorer des souvenirs sans qu'il y ait stimulation sensorielle au préalable. Quant au récepteur, les élèves n'ont pas assimilé la totalité de ses propriétés pour dominer réellement ce concept tel qu'il est défini en cours. De même, il apparait qu'après le cours, le concept de codage de l'information n'est assimilé que par une minorité d'élèves. Seul le concept de réflexe semble approprié par la majorité des élèves.

Ainsi, la présence chez la majorité des élèves de Terminale de certaines déficiences qui avaient été mises en évidence au niveau de l'acquisition de certains concepts définis dans le cours de Seconde, n'ont pu être comblées par le cours de biologie de Terminale qui pourtant s'appuie sur ces mêmes concepts.

30 Le problème posé par l'enseignement du système nerveux au lycée est donc de trouver une réponse aux questions suivantes: pourquoi les élèves présentent-ils autant de 
difficultés pour acquérir les concepts qui sont définis dans le cours? Pourquoi considèrent-ils majoritairement l'organisme comme une boite noire? Pourquoi ont-ils une telle méconnaissance des structures biologiques et de leur fonctionnement?

Tenter, dans un premier temps, de répondre à ces questions, puis, dans un deuxième temps, d'élaborer les moyens didactiques permettant l'éradication de ces problèmes, sont donc les enjeux auxquels doit faire face l'enseignement du système nerveux. Quoi enseigner exactement, sous quelle forme, avec quels outils pédagogiques sont autant de points à déterminer pour que les élèves arrivent à s'approprier un tel ensemble de connaissances et qu'ils parviennent à les mobiliser, à les utiliser, à les transférer, soit pour résoudre certains problèmes que leur pose la vie courante, soit pour mieux cerner les phénomènes qui sont inhérents au fonctionnement de leur propre corps. Car fondamentalement, un des buts primordiaux que doit se fixer ce type d'enseignement n'est-il pas de favoriser la connaissance qu'ont les élèves d'eux-mêmes?

\section{BIBLIOGRAPHIE}

CLEMENT P., « Conceptions sur le cerveau, santé et normalisation », in La philosophie du remède. Champ Vallon, 1993. pp. 152-172.

CLEMENT P., « difficile évolution des conceptions sur les rapports entre cerveau, idées et âme » in : Giordan A., Girault Y. \& Clément P., Conceptions et connaissances, Berne, Suisse, Peter Lang, 1994, pp. 73-91.

DARLEY B., L'enseignement de la démarche scientifique dam les travaux pratiques de biologie à l'université. Analyses et propositions., Thèse de Doctorat, Université de Grenoble 1, France, 1994. OUVRAGE COLLECTIF - (MAFPEN). Séquences centrées sur un obstacle, biologie, géologie. Collection Démarches Innovantes, CNDP. 1994. 108 p.

MEIN M.T., «Les représentations du cerveau : modèles historiques ", in Aster, $\mathrm{N}^{\circ} 7$, Paris, INRP. 1988, pp. 185-204.

MEIN M.T. \& CLEMENT P., « Comment se représente-t-on aujourd'hui notre cerveau ? », in Actes des Dixièmes Journées sur l'Enseignement Scientifique de Chamonix, Paris, LIREST. Université Paris VII. 1988, pp. 243-252.

\section{RÉSUMÉS}

$\mathrm{Au}$ cours de cette étude, nous avons inféré, avant et après le cours, les conceptions que présentent des élèves de Terminale au sujet de certains concepts dont les définitions sont introduites lors de l'enseignement du système nerveux. Globalement il ressort que le cours de Terminale n'a que peu d'influence sur la nature des conceptions des élèves. Majoritairement construites de façon empirique, elles présentent un caractère fortement contextualisé au dispositif des 1ers apprentissages. 
Over the course of this study, we inferred what, the conceptions of "terminale" year "lycée" student were, both at the beginning and at the end of Bach class session, on the subject of certain biology concepts for which definitions are introduced when the nervous system is treated in class. Essentially speaking, results show that the class session bas only a slight influence upon the nature of the students' conceptions. These conceptions, for the must part, empirically constructed, are shown to be, essentially speaking, highly contextualized in relation to previous school learning.

INDEX

Mots-clés : conception, didactique, système nerveux

Keywords : conception of (...), didactic, nervous system

\section{AUTEURS}

JEAN-LOUIS BEC

Laboratoire de modélisation de la relation pédagogique, équipe ERES, université de Montpellier II DANIEL FAVRE

Laboratoire de modélisation de la relation pédagogique, équipe ERES, université de Montpellier II 